\title{
Percepción de actitudes nocivas en el uso de las redes sociales en los jóvenes adolescentes
}

\section{Perception of harmful attitudes in the use of social networks in teenagers}

\author{
Cristina Sánchez Romero \\ UNED. Facultad de Educación (España) \\ csanchez@edu.uned.es \\ M. ㄹ Carmen López Berlanga \\ Colegio López Vicuña (España) \\ maricarmen@lopezvicu.es
}

Recibido: 03/11/2019

Aceptado: $23 / 03 / 2020$

Publicado: 01/06/2020

\section{RESUMEN}

Los jóvenes adolescentes se encuentran inmersos en las redes sociales a través de sus dispositivos móviles. El objetivo de la investigación se centra en analizar la percepción de actitudes nocivas entre los jóvenes adolescentes en las redes sociales. Para ello, se han seleccionado una muestra de 202 estudiantes del nivel educativo de secundaria obligatoria a quienes se les ha aplicado un cuestionario mixto (alfa 0.78 ) del que se ha seleccionado las unidades de información cualitativa de la narrativa de los informantes claves. Los resultados muestran que los estudiantes han percibido agresiones a través de insultos y amenazas por compartir archivos fotográficos (46.03\%) en las principales redes sociales como son Instagram (61.4 \%) y WhatsApp (26.2\%). Estas agresiones que sufren los estudiantes se pueden solventar si se desarrolla una formación en la inclusión digital desde edades tempranas. Esto permitirá un uso adecuado de los dispositivos móviles y una comunicación e intercambio de información en las redes sociales desde actitudes positivas. Se requiere una inclusión digital de los estudiantes para el uso crítico de los archivos que comparten a través de las redes sociales.

\section{PALABRAS CLAVE}

Actitudes; jóvenes adolescentes; redes sociales; inclusión digital

\section{ABSTRACT}

The teenagers are immersed in social networks through their mobile devices. The objective of the research focuses on analyzing the perception of harmful attitudes among young adolescents in social networks. To do this, a sample of 202 students of compulsory secondary education level have been selected, to whom a mixed questionnaire (alpha 0.78 ) has been applied, from which the qualitative information units of the narrative of the key informants have been selected. The results show that students have perceived aggressions through insults and threats by sharing photographic files $(46.03 \%)$ in the main social networks such as Instagram (61.4\%) and WhatsApp (26.2\%). These aggressions suffered by students can be solved if a training in digital inclusion is developed from an early age. This will allow an adequate use of mobile devices and a communication and exchange of information in social networks from positive attitudes. It requires a digital inclusion of students for the critical use of the files they share through social networks.

\section{KEYWORDS}

Attitudes; young adolescents; social networks; digital inclusion 


\section{CITA RECOMENDADA}

Sánchez, C. \& López, M.C. (2020). Percepción de actitudes nocivas en el uso de las redes sociales en los jóvenes adolescentes. Revista Interuniversitaria de Investigación en Tecnología Educativa, 8, 1-13. http://dx.doi.org/10.6018/riite.401801

\section{Principales aportaciones del artículo y futuras líneas de investigación:}

- Analizar las actitudes de los menores y los jóvenes en el uso de las redes sociales.

- Identificar actitudes nocivas en la convivencia digital como prevención del ciberbullying.

- Investigar la Identidad digital en el uso de las redes sociales.

\section{INTRODUCCIÓN}

En la sociedad actual las tecnologías han hecho que nos adaptemos a nuevas formas tanto de formación como de información y comunicación a través de las tecnologías (Cabero, et ál. 2017; Salinas, 2004) este escenario, se ha observado el gran impacto que han producido las redes sociales en la población (Statista $\mathrm{GmbH}, 2016$ ). Por ello, principalmente nos preocupa el uso prematuro de los dispositivos móviles entre los menores y jóvenes adolescentes; y las actitudes nocivas que se desencadenan a través de los mismos, no sólo en la comunicación e intercambio de la información en el entornos de las redes sociales sino en las diferentes manifestaciones que emergen en un contexto digital (Sánchez-Romero y Álvarez-González, 2018)

Esta preocupación nos lleva a preguntarnos sobre el uso que hacen los adolescentes en diferentes redes sociales y las actitudes que muestran a través de la mensajería instantánea entre iguales. Para ello, nos hemos centrado en investigaciones realizadas sobre el elevado uso de los dispositivos móviles y los Smartphone por los jóvenes de nuestro país. Las estadísticas de estas investigaciones muestran que la utilización de las tecnologías principalmente Smartphone- comienza a los 10 años, siendo de 10 a 15 años el período en el que aumenta su utilización (Instituto Nacional de Tecnologías de la Comunicación y France Telecom España, 2010; INTECO y Orange, 2011), por ello, desde una perspectiva formativa se presume la necesidad de una formación temprana en el uso crítico de los dispositivos móviles.

La mayoría de los estudios muestran que principalmente los estudiantes utilizan las redes sociales para fines de ocio (Sánchez-Vera, et al, 2011). Por tanto, nos hemos planteado cómo son las actitudes de los adolescentes en las redes sociales en el contexto personal de los estudiantes. Para ello, hemos analizado diferentes investigaciones en las que se confirma la vulnerabilidad de los jóvenes en éste, contexto y cómo también se generan otros problemas como las adicciones (Echeburúa y De Corral, 2010) y, las actitudes nocivas que pueden terminar en conductas de acoso en la red (Garaigordobil, 2015). Estas variables analizadas hacen que el manejo de las redes sociales no sea el adecuado (Hinduja y Patchin, 2010).

En ocasiones, por un lado, estas actitudes se generan por desconocimiento y desinformación del buen uso de la información y comunicación en la red (Foshee et al., 2016; Mossop, 2012) y, por otro, se pueden desarrollar de forma intencionada entre iguales (Kowalski y Limber, 2013) transformándose en actitudes cibernéticas intimidadoras, en intercambio de fotografías sin previo permiso y, generando hilos de conversación y comentarios perjudiciales hacia otras personas víctimas de dichas acciones (Wang, lannotti, y Nansel, 2009).

Por tanto, ante esta problemática nos plateamos analizar el uso que hacen los estudiantes de educación secundaria de las redes sociales y que actitudes perciben de las mismas en la línea de investigaciones anteriores (Sánchez-Romero y Álvarez-González, 2018). 


\subsection{Las redes sociales}

Siguiendo la definición de Morduchowicz et al., 2010 sobre las redes sociales como comunidades virtuales que agrupan a personas que se relaciona entre sí y comparten información e intereses comunes. Nos planteamos las actitudes de esas personas en el uso de las principales redes sociales y, el tipo de información que comparten, particularmente para este estudio los estudiantes de secundaria que forman parte de diferentes redes sociales y grupos de mensajería instantánea.

Principalmente nos preocupa el cambio de conductas y la emergente actitud negativa en los comentarios e intercambios de información que se desarrollan en este escenario virtual.

Autores como Bernete (2010) describen el cambio de los jóvenes en sus relaciones sociales y su repercusión en las interacciones con otros agentes sociales como la familia, los docentes y los compañeros en las redes sociales.

Por tanto, se destaca la necesidad de profundizar en los cambios que se están perfilando en los hábitos online de los adolescentes, por la gran incidencia en su vida cotidiana y las implicaciones socioculturales (Klein, 2006). Este autor analiza las prácticas online de los adolescentes entre 12 y 17 años en Red destacando el uso más intensivo del tiempo y en la utilización de las redes sociales -principalmente aquellas que les permiten mantener contacto y compartir contenidos- entre iguales.

Por ello, ante un análisis de las principales redes sociales que utilizan esta población estudiantil nosotros hemos seleccionado las siguientes redes sociales en las que los estudiantes comparten información e interaccionan en las mismas:

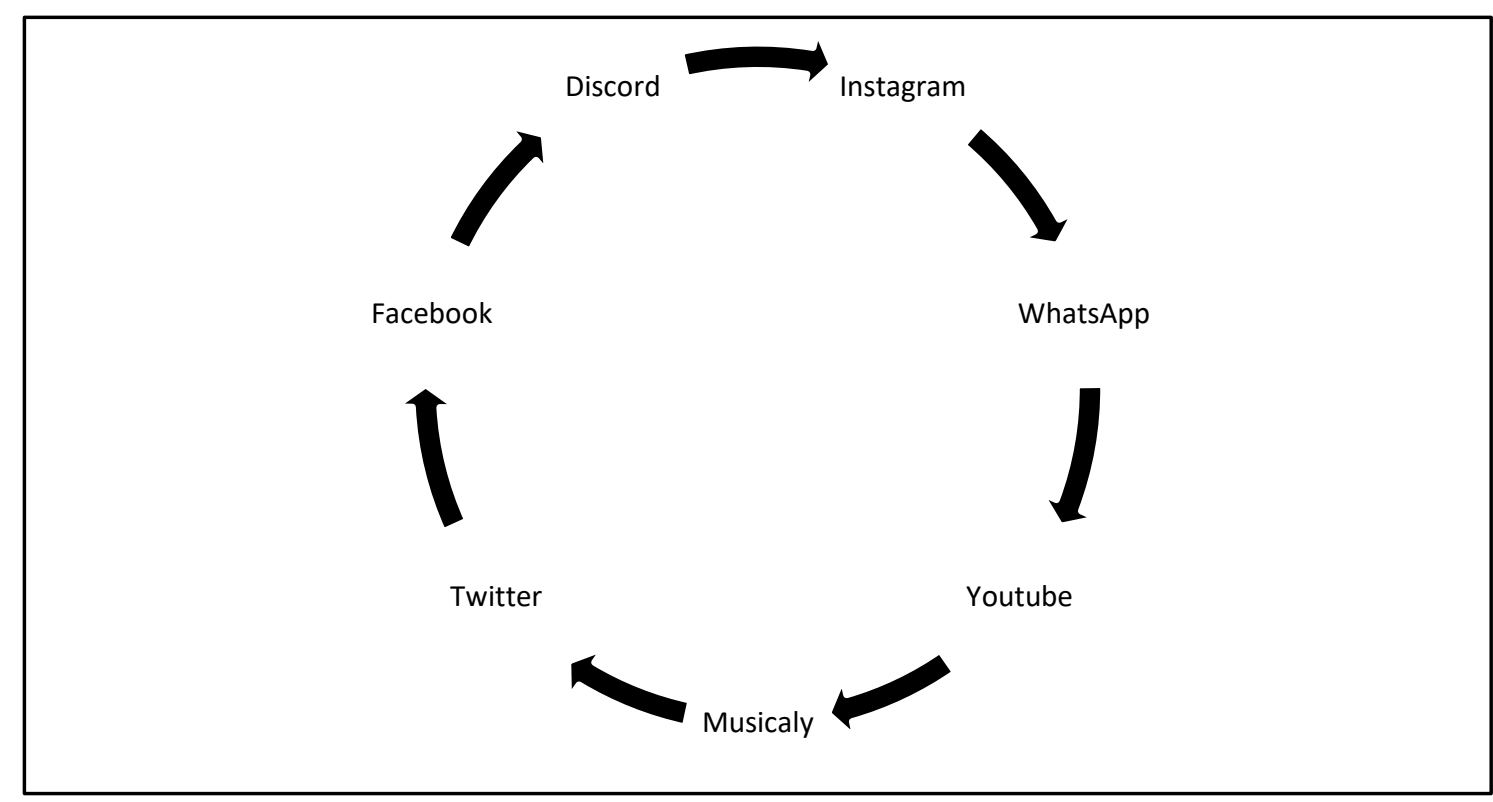

Figura 1. Principales Redes Sociales utilizadas por los Adolescentes. Fuente "We are Social" junto con Hootsuite (2018)

En las principales redes seleccionadas el número de usuarios ha ido incrementando en los últimos años. Estos datos se reflejan en el informe, publicado por "We are Social" junto con Hootsuite (2018) en el que vemos los siguientes datos:

- Facebook. Esta red siguiendo las estadísticas propias de la compañía (Facebook, 2016) tiene más de 936 millones de usuarios activos diarios y más de 1.44 millones de usuarios activos mensuales. Desatacan en ésta estadística datos como que 1.25 millones de usuarios están activos móviles mensuales en diferentes países, principalmente fuera de Estados Unidos y Canadá. 
- Twitter. Sobre los usuarios de Twitter podemos destacar según las estadísticas de la compañía (Twitter, 2014) que tiene más de 241 millones de usuarios activos mensuales, y que se envía por día más de 500 millones de tweets. También podemos observar que la mayoría de los usuarios activos de Twitter están en el móvil (77 \%) y que también las cuentas están fuera de Estados unidos. Además, Twitter admite más de 35 idiomas. En el mes de julio 2018 se cuenta con más de 336 millones de usuarios, por lo que se refleja un gran aumento desde el dato obtenido del 2014.

- YouTube Respecto a las estadísticas en el Canal de YouTube (YouTube, 2016) destacamos el intercambio de archivos de vídeos subidos, más de 78.3 millones de vídeos subidos por día. En julio del 2018 tenía más de 1900 millones de usuarios, aumento considerable desde los usuarios registrados en el 2016.

Discord. El uso de las apps se ha ido incrementando en los últimos años llegando al $111 \%$ según informe Ditrendia (2018). A través de Discord según informe de VentureBeat en 2018 (Takahashi, 2018) actualmente se mandan alrededor de 200 millones de mensajes cada día y hay unos 8'9 millones de usuarios activos a diario.

- WhatsApp. Es el servicio de mensajería más utilizada en todo el mundo tenía 1200 millones de usuarios activos (2017). En el 2018 se incrementa con más de 1500 millones de usuarios activos en un mes. Consiguiendo que se lleguen a intercambiar al día más de 60.000 millones de mensajes.

- Instagram. Conocida como Red social de fotografía más popular en el mundo, más de 40.000 millones de fotografías compartidas durante todo el año y más de 95 millones de fotos y vídeos compartidos al día (Sloan, et al, 2017; Yuan, 2018). En el 2017 contaba con más de 600 usuarios en activo, en 2018 con más de más de 1.000 millones de usuarios activos en un mes, consiguiendo duplicar en un año el número de usuarios activos.

- Musically. Conocido actualmente con el nombre de Tik Tok Red social fundada en 2016 de origen chino, en la que los usuarios pueden crear videos de 15 segundos de duración a los que unen un clip musical con distintos efectos y filtros. En el año 2018337 millones de usuarios se descargaron la aplicación desde Google Play e iPhone. En la actualidad supera los 500 millones de usuarios activos en todo el mundo (2018).

Se puede observar en todas las redes que el incremento de participantes es emergente. Nosotros destacamos Facebook, Instagram y WhatsApp como aquellas más utilizadas por los jóvenes adolescentes en este estudio y en estudios anteriores (Sánchez Romero \& Álvarez González, 2018). Estas redes forman parte de su día a día y, en ellas comparten principalmente sus estados e intercambian información.

\subsection{Relación del uso de las redes sociales con los adolescentes}

Los adolescentes utilizan las redes sociales como medio de comunicación y de interacción social (IAB Spain, 2017). Las redes sociales les permiten una comunicación rápida e instantánea por medio de mensajes fotos o vídeos lo que llega a convertirse en una necesidad y rutina diaria. Esta necesidad siguiendo la clasificación de Del Moral (2005) puede ser de cuatro tipos: mantener amistades, crear amistades, ocio y entretenimiento, y gestión interna de organizaciones empresariales.

La identidad digital se modela a través del uso y participación en las redes (Boyd, 2007), en la identidad se refleja los perfiles públicos de los adolescentes en los que se advierten según Vanderhoven, et al., (2014) la emergente preocupación por la seguridad y privacidad en las mismas.

Nuestra preocupación se genera en esta línea, en la necesidad de crear entornos seguros del uso de los dispositivos móviles en contextos vulnerables (Sánchez-Romero, 2014) y en el estudio de los comportamientos de los adolescentes dentro de las redes sociales, avalada por estudios como los de Utz. S Tanis y Vermeulen (2012) que analizan la actitud del comportamiento del adolescente, ligada al propósito que estos tienen de alcanzar prestigio dentro de las mismas (número de seguidores, likes recibidos, etc.) y, también a la estrecha 
relación entre sus características fuera de las redes sociales (Ahn, 2012) o en la comunidad interpersonal cotidiana de los adolescentes (Courtois, et al, 2012).

De ahí, la importancia de analizar los riesgos que se pueden percibir en las redes. Los estudiantes pueden estar sometidos a situaciones de acoso en los que su intimidad y privacidad son más vulnerables (Livingstone, 2018). Por ello, describimos a continuación el uso que hacen los estudiantes de secundaria en las redes sociales, y cómo ellos perciben las actitudes de sus iguales.

\section{MÉTODO}

\subsection{Objetivo/hipótesis}

El objetivo principal de esta investigación es analizar el uso que hacen los estudiantes de secundaria de las redes sociales y que actitudes perciben en las mismas.

Entre los objetivos específicos destacamos:

- Analizar el tipo de redes que utilizan los jóvenes y menores adolescentes.

- Considerar la edad con la que se inician en el mundo de las redes sociales.

- Identificar las actitudes negativas en la comunicación en las redes que pueden contribuir a problemas de acoso en la red.

\subsection{Metodología / Método}

Desde un enfoque mixto en la investigación, hemos seleccionado la parte cualitativa de la misma para este estudio en la línea de (McMillan y Schumacher, 2005; Peter, 2000; Taylor y Bogdan, 1987) y para la obtención de la narrativa de informantes claves en la línea de autores como Clandinin y Huber (2010). Con esta metodología queremos reflejar como son los estudiantes de los niveles educativos de Educación Secundaria Obligatoria en cuanto al uso de las redes sociales y su percepción en actitudes nocivas desde su propia percepción, sujetos claves en el proceso.

\subsubsection{Población}

La población seleccionada para este estudio han sido estudiantes de $1 .^{\circ}$ a $4 .^{\circ}$ de educación secundaria. La zona demográfica seleccionada para el estudio se encuentra en la zona Este de Madrid.

\subsubsection{Muestra}

La muestra no probabilística ha sido de 202 estudiantes de edades comprendidas entre (12 y 15 años) con una media de edad de 13.5 años. De esta participación un $42.1 \%$ pertenecen al género masculino y un $57.9 \%$ al femenino.

\subsubsection{Instrumento}

El instrumento para la recogida de datos que se ha utilizado ha sido elaborado y adaptado (ver Anexo I) de la dimensión IV (ver Anexo II) titulada Relaciones Escolares: "apartado social" de la P56 y PP7 constituido por 11 Ítems cada una, donde se analizan la frecuencia si han sufrido alguna agresión o la frecuencia en la que han visto la agresión hacia de Ruiz-Ramírez (2013); Ruiz-Ramirez (et al.) (2017). Ambos aspectos relacionados y enfocados al tema de interés de este estudio y adaptado a la muestra de nuestro país. Su estructura es ad hoc con preguntas de escala tipo Likert, preguntas dicotómicas y preguntas abiertas, enfocadas analizar en este estudio dos factores: el uso y percepción de las actitudes en las Redes Sociales (Ver tabla 1) y su relación con el factor edad. Obteniendo un alfa de Cronbach de 0.78. 
Tabla 1. Preguntas analizadas que se han seleccionado para este estudio.

\begin{tabular}{ll}
\hline PA4 & Red que más utilizas con tus amigos \\
\hline PA6 & Percepción de actitudes nocivas recibidas a tus compañeros \\
\hline
\end{tabular}

\subsubsection{Recogida de datos}

Se han realizado de forma presencial en una Institución Educativa, realizada de forma voluntaria y anónima confidencial y para fines de investigación de acuerdo con la Ley Orgánica $3 / 2018$ de 5 de diciembre, de protección de datos personales y garantía de los derechos digitales.

\subsubsection{Herramienta para el análisis}

El análisis de los datos se ha realizado a través del programa SPSS 21.0 (para las preguntas de corte cuantitativo) y el programa Atlas ti para las narrativas obtenidas de los estudiantes en las preguntas abiertas (preguntas de corte cualitativo).

\section{RESULTADOS}

Analizados los datos a continuación, mostramos los resultados obtenidos:

A la pregunta ¿Cuál es la red que más utilizas con tus amigos?

Los resultados muestran que la red más utilizada por los estudiantes es Instagram con $61.4 \%$ y WhatsApp con $26,2 \%$ (ver tabla 2 ).

Tabla 2. Red que más utilizas con tus amigos

\begin{tabular}{llcccc}
\hline & & Frecuencia & Porcentaje & $\begin{array}{c}\text { Porcentaje } \\
\text { válido }\end{array}$ & $\begin{array}{c}\text { Porcentaje } \\
\text { acumulado }\end{array}$ \\
\hline Válido & Perdidos & 2 & 1,0 & 1,0 & 1,0 \\
& Instagram & 124 & 61,4 & 61,4 & 62,4 \\
& WhatsApp & 53 & 26,2 & 26,2 & 88,6 \\
YouTube & 11 & 5,4 & 5,4 & 94,1 \\
& Musicaly & 6 & 3,0 & 3,0 & 97,0 \\
& Discord & 1 &, 5 &, 5 & 97,5 \\
Facebook & 3 & 1,5 & 1,5 & 99,0 \\
Twitter & 2 & 1,0 & 1,0 & 100,0 \\
Total & 202 & 100,0 & 100,0 & \\
\hline
\end{tabular}

Fuente: Datos de investigación

Ambas redes se caracterizan por el intercambio de archivos fotográficos y comentarios sobre los mismos. Por ejemplo, en Instagram los usuarios toman fotos con sus dispositivo móviles y suben las mismas, esta aplicación se ha convertido en una de las herramientas de redes sociales más populares (Sloan et al., 2017).

A la pregunta ¿Qué redes sociales utilizas?

Los resultados obtenidos nos indican que la red social más utilizada por los jóvenes es el WhatsApp e Instagram, como se puede observar en la Tabla 3. y su uso va en aumento a la vez que se va avanzando en edad (ver figura 2 ). 
Tabla 3. Edad y Red social que utilizas

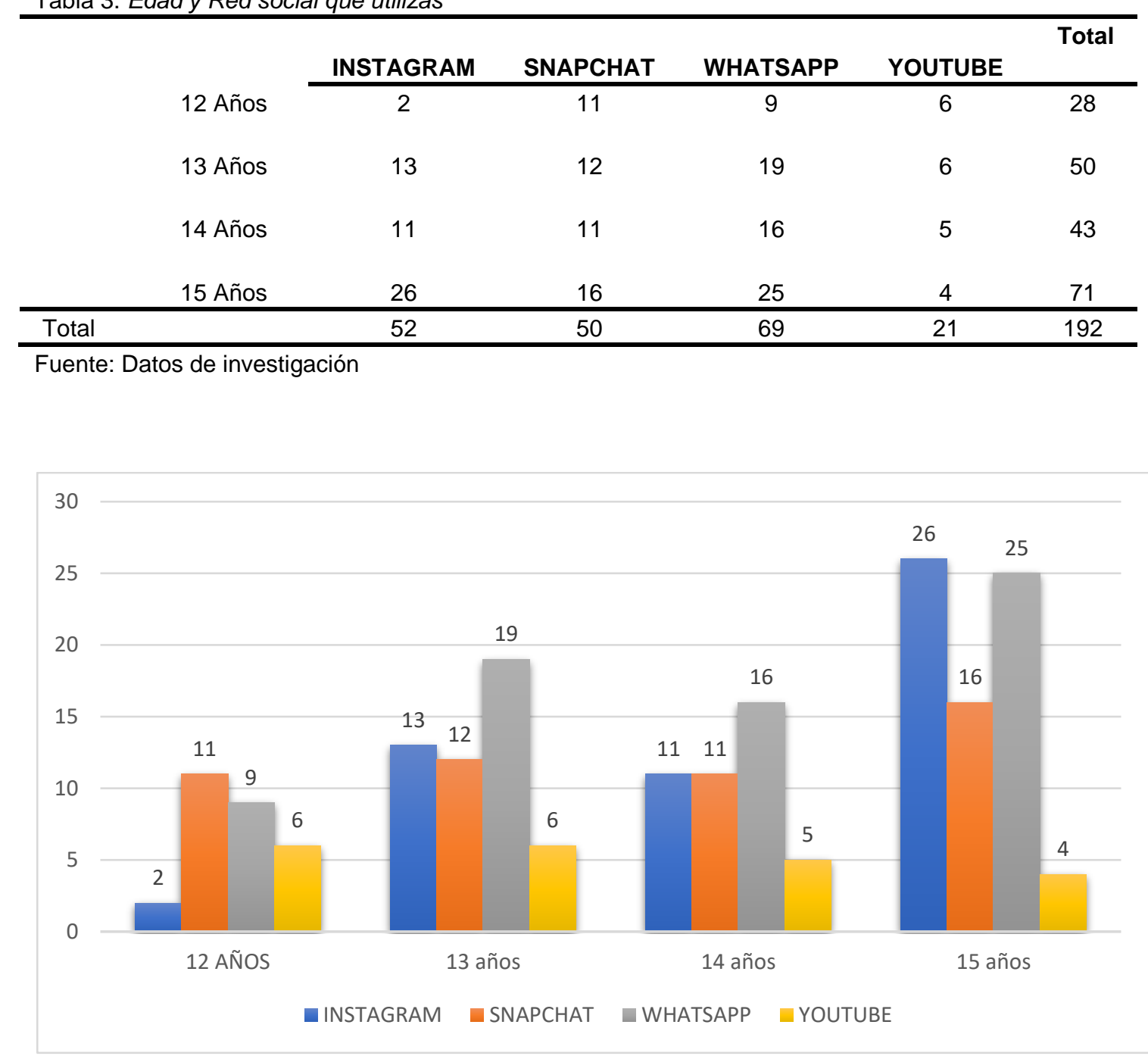

Figura 2: Redes sociales que utilizan según edad.

Se puede observar en la Figura 2 que el uso de WhatsApp va en aumento a la vez que se va avanzando en edad. Esto también ocurre con Instagram.

Si relacionamos las redes sociales más utilizadas con la edad, podemos observar que ambas redes destacan sobre las demás, pero hay una variación en la edad. Lo que nos indica que el uso de las redes va incrementando según avanzan las edades (Ver tabla 3 y figura 2).

Referente a la pregunta realizada a los estudiantes si han percibido que otras personas de la red que comparten han recibido algún tipo de actitud nociva. Los resultados obtenidos se observan en la Tabla 4.

Tabla 4. Percepción de actitudes nocivas recibidas a tus compañeros.

\begin{tabular}{llcccc}
\hline \multicolumn{1}{c}{} & & & & Porcentaje \\
& & Frecuencia & Porcentaje & Porcentaje válido & acumulado \\
\hline Válido & $\mathrm{Si}$ & 93 & 46,0 & 46,5 & 46,5 \\
& No & 107 & 53,0 & 53,5 & 100,0 \\
& Total & 200 & 99,0 & 100,0 & \\
Perdidos & Sistema & 2 & 1,0 & & \\
& & & & & \\
\hline Total & 202 & 100,0 & & \\
\hline
\end{tabular}

Fuente: Datos de investigación 
La respuesta ha sido positiva en un $46 \%$ de los estudiantes encuestados. Esto indica que sí se perciben actitudes nocivas entre iguales. La mayoría de los estudiantes consultados coinciden en que han recibido insultos y amenazas a través de las redes sociales. Esto se debe principalmente cuando han subido y compartido una fotografía personal. Describen que "les han insultado y amenazado a través de la red social compartida por subir y/o compartir algún archivo fotográfico". También en el $46 \%$ de los estudiantes, destacan las opiniones de divulgar algún tipo de información falsa, conocidas actualmente como Fake new.

Por tanto, tras los análisis realizados podemos confirmar el aumento del uso de los dispositivos móviles y la alta participación en las redes sociales entre los adolescentes.

Los resultados muestran que los estudiantes de secundaria obligatoria utilizan las redes sociales a través de sus dispositivos móviles para el ocio, la comunicación y el intercambio de información desde edades tempranas.

Entre las redes más utilizadas destacan las aplicaciones para subir fotos como Instagram y las de mensajería instantánea como WhatsApp. Por ejemplo, en Instagram los usuarios toman fotos con sus dispositivo móviles y suben las mismas, esta aplicación se ha convertido en una de las herramientas de redes sociales más populares (Sloan et al., 2017). También la mensajería instantánea a través de WhatsApp como muestran estudios de Montag et al. (2015) contribuye al uso excesivo de los teléfonos inteligentes en los jóvenes adolescentes.

\section{DISCUSIÓN Y CONCLUSIONES}

Las principales redes que utilizan son aquellas en las que comparten vivencias personales y suelen intercambiar archivos personales como fotografías y vídeos. Los estudiantes hacen de las redes sociales un medio de comunicación, de ocio y de intercambio de información sin conocer los principales riesgos que pueden sufrir, en los que podemos destacar situaciones de acoso y ciberbullying (Buelga, et. al, 2010).

Según avanzan en edad utilizan un mayor número de redes sociales. En este sentido, se requiere una inclusión digital y crítica para la correcta utilización de los dispositivos y redes sociales (Sang, et al, 2011). Debemos trasferir a nuestros estudiantes la competencia digital para el uso socioeducativo de las redes sociales de forma que no se encuentren en un entorno vulnerable sino en un entorno seguro en el que puedan intercambiar su información a través de los medios digitales (Stald, 2008)

Respecto a la percepción de actitudes nocivas a otros compañeros, los resultados muestran que sí existen estas actitudes nocivas entre iguales mostradas en comentarios peyorativos sobre la persona que sube y/o comparte un archivo. Los estudiantes observan estas actitudes en sus mismas redes pero no lo comunican (Del Río, et al, 2010). Por tanto, surge la necesidad de enseñar y potenciar el uso de las redes (Coll, 2009).

Para ello, consideramos muy importante desarrollar pautas para la creación de entornos seguros en internet desde edades tempranas desde una perspectiva interdisciplinar (Sánchez y Álvarez, 2017) y desarrollando una plena formación para la integración y uso de las TIC en diferentes contextos socioeducativos (Del Río, et al., 2010; Echeburúa \& De Corral, 2010; Marcelo, 2002; Moreira, 2008; Sánchez \& Álvarez,2017).

Por tanto, es muy importante analizar la participación de los adolescentes en las Redes Sociales. Esto ha de implicar a toda la Comunidad educativa para evitar actitudes nocivas y agresiones entre iguales en las redes sociales (Foshhe et al, 2016).

Nos planteamos nuevas formas de alfabetización digital para los jóvenes adolescentes en la línea de las investigaciones realizadas por Greenhow \& Robelia (2009) encaminada a desarrollar un aprendizaje social en las mismas.

\section{5.- ENLACES}

Instrumento de recogida de datos: Se ha realizado una adaptación de la dimensión del cuestionario de Ruiz-Ramírez (2013). Depositado en el repositorio Nacional de México con el 
siguiente enlace https://bit.ly/3fwUIYE . También se ha recogido información de las Tablas de Análisis basadas en el cuestionario citado del artículo Ruiz-Ramírez, R.; Sánchez-Romero, C.; Zapata-Martelo, E.; García-Cué, J. L.; Pérez-Olvera, Ma. A.; Martínez-Corona, B. \& RojoMartínez, G. (2017). Manifestaciones del bullying en la Preparatoria Agrícola. Universidad Autónoma de Chapingo, México. Revista Latinoamericana de Ciencias Sociales, Niñez y Juventud, 15 (2), pp. 1149-1163. DOI:10.11600/1692715x.1522425112016

\section{REFERENCIAS BIBLIOGRÁFICAS}

Ahn, J. (2012). Teenagers and social network sites: Do off-line inequalities predict their online social networks? First Monday, 17(1). https://doi.org/10.5210/fm.v17i1.3752

Bernete, F. (2010). Usos de las TIC, Relaciones sociales y cambios en la socialización de las y los jóvenes. Revista de Estudios de Juventud, 88, 97-114. https://bit.ly/2yAjfeM

Boyd, D. (2007). Why youth (heart) social network sites: The role of networked publics in teenage social life. SSRN. https://bit.ly/3dru6qa

Buelga, S., Jesus Cava, M. \& Musitu, G. (2010). Cyberbullying: victimizacion entre adolescentes a traves del telefono movil y de internet. Psicothema, 22(4), 784-789. https://bit.ly/369N3v5

Cabero J., Fernández, B., y Marín, V. (2017). Dispositivos móviles y realidad aumentada en el aprendizaje del alumnado universitario. RIED. Revista Iberoamericana de Educación a Distancia, 20(2), 167-185. https://doi.org/10.5944/ried.20.2.17245

Clandinin, D. J., \& Huber, J. (2010). Narrative inquiry. In B. McGaw, E. Baker \& P.P. Peterson (Eds.), International Encyclopedia of Education (pp. 436-441). Elsevier. https://doi.org/10.1016/B978-0-08-044894-7.01387-7

Coll, C. (2008). Aprender y enseñar con las TIC: expectativas, realidad y potencialidades. Boletín de la institución libre de enseñanza, 72(1), 7-40. https://bit.ly/35SNjPi

Courtois, C., All, A., \& Vanwynsberghe, H. (2012). Social network profiles as information sources for adolescents' offline relations. Cyberpsychology, Behavior, and Social Networking, 15(6), 290-295. https://doi.org/10.1089/cyber.2011.0557

Del Río, J., Sádaba, C., \& Bringue, X. (2010). Menores y redes ¿sociales?: de la amistad al cyberbullying. Revista de Estudios de Juventud, 88, 115-129. https://bit.ly/2xO3/l6

Ditrendia. (2016). Informe Mobile en España y en el Mundo. Amic. https://bit.ly/3ce89Ld

Echeburúa, E., \& De Corral, P. (2010). Adicción a las nuevas tecnologías y a las redes sociales en jóvenes: un nuevo reto. Adicciones, 22, 91-96. https://doi.org/10.20882/adicciones.196

Greenhow, C., \& Robelia, B. (2009). Old communication, new literacies: Social network sites as social learning resources. Journal of Computer-Mediated Communication, 14(4), 11301161. https://doi.org/10.1111/j.1083-6101.2009.01484.x

Hinduja, S., \& Patchin, J. (2010). Bullying, Ciberbullying, and Suicide. Archives of Suicide Research, 14(3), 206-221. https://bit.ly/2SMkNcO

IAB Spain. (2017). Estudio Anual Redes Sociales. Iabspain. https://bit.ly/3dtlqPc

Kamel Boulos, M. N., Giustini, D. M., \& Wheeler, S. (2016). Instagram and WhatsApp in health and healthcare: An overview. Future Internet, 8(3), 37. https://doi.org/10.3390/fi8030037

Klein, J.T. (2006). A Platform for a Shared Discourse of Interdisciplinary Education. Journal of Social Science Education, 5(4), 10-18. https://bit.ly/2ACbi9D

Livingstone, S., Mascheroni, G. \& Staksrud, E. (2018). Investigación europea sobre el uso de internet para niños: evaluar el pasado y anticipar el futuro. New Media y Society, 20(3), 1103-1122.

Marcelo, C. (2002). Los profesores como trabajadores del conocimiento: Certidumbres y 
desafíos para una formación a lo largo de la vida. Educar, 30, 27-56. https://bit.ly/3ftGAiY

McMillan, J. H., \& Schumacher, S. (2005). Investigación educativa una introducción conceptual. Pearson educación. https://bit.ly/2yd993x

Montag, C., Błaszkiewicz, K., Sariyska, R., Lachmann, B., Andone, I., Trendafilov, B., Eibes M. \& Markowetz, A. (2015). Smartphone usage in the 21st century: Who is active on WhatsApp? BMC Research Notes, 8(1): 331. https://doi.org/10.1186/s13104-015-1280-z

Morduchowicz, R., Marcon, A., Sylvestre, V. \& Ballestrini, F. (2010). Los adolescentes y las redes sociales. Escuelas y Medios. Ministerio de Educación de la Nación de Argentina.

Moreira, M. A. (2008). La innovación pedagógica con TIC y el desarrollo de las competencias informacionales y digitales. Investigación en la escuela, 64. 5-17. https://bit.ly/2YWicRt

Peter, A. (2000). Métodos cualitativos de investigación. Estudios Pedagógicos (Valdivia), 26, 91-106. http://dx.doi.org/10.4067/S0718-07052000000100007

Ruíz-Ramírez, R. (2013). La deserción escolar en la educación rural [tesis de maestría no publicada, Universidad de México]. Repositorio nacional https://www.repositorionacionalcti.mx/recurso/oai:colposdigital.colpos.mx:10521/2147

Ruiz-Ramírez, R.; Sánchez-Romero, C.; Zapata-Martelo, E.; García-Cué, J. L.; Pérez-Olvera, Ma. A.; Martínez-Corona, B. \& Rojo-Martínez, G. (2017). Manifestaciones del bullying en la Preparatoria Agrícola. Universidad Autónoma de Chapingo, México. Revista Latinoamericana de Ciencias Sociales, Niñez y Juventud, 15 (2), pp. 1149-1163. http://www.scielo.org.co/pdf/rlcs/v15n2/v15n2a25.pdf

Salinas, J. (2004). Cambios metodológicos con las TIC. Estrategias didácticas y entornos virtuales de enseñanza-aprendizaje. Bordón, 56(3-4), 469-481. https://bit.ly/2WffLaQ

Sánchez Romero, C., \& Álvarez González, E. (2018). Actitudes nocivas y riesgos para los menores a través de los dispositivos móviles. Revista de Estudios y Experiencias en Educación, 2(2), 147-161 https://doi.org/10.21703/rexe.especial3 201814716113

Sánchez-Vera, M. del M., Prendes-Espinosa, M. P., \& Serrano-Sánchez, J. L. (2011). Modelos de interacción de los adolescentes en contextos presenciales y virtuales. Edutec. Revista Electrónica De Tecnología Educativa, 35: a158. https://bit.ly/3fwJmns

Sloan, L., \& Quan-Haase, A. (Eds.). (2017). The SAGE handbook of social media research methods. SAGE. https://bit.ly/35HRgWN

Stald, G. (2007). Mobile identity: Youth, identity, and mobile communication media. In D. Buckingham, Youth, Identity, and Media (pp. 143-164). MacArthur Foundation Digital Media and Learning Initiative. https://bit.ly/3g3cwec

Statista GmbH. (2016). El portal de estadísticas. https://bit.ly/3bQ7heP

Taylor, S.J. \& Bogdan, R. (1987). Introducción a los métodos cualitativos de investigación: la búsqueda de significados. Paidós. https://bit.ly/2zIXHwR

Vanderhoven, E., Schellens, T., Valcke, M., \& Raes, A. (2014). How safe do teenagers behave on facebook? An observational study. PLOS ONE, 9(8). https://doi.org/10.1371/journal.pone.0104036

Takahashi, D. (2018). Newzoo: Smartphone users will top 3 billion in 2018, hit 3.8 billion by 2021. Venturebeat. https://bit.ly/2YK1moM

Wang, J., lannotti, R. J., \& Nansel, T. R. (2009). School Bullying Among Adolescents in the United States: Physical, Verbal, Relational, and Cyber. Journal of Adolescent Health, 45(4), 368-375. https://doi.org/10.1016/j.jadohealth.2009.03.021

Yuan, L. (2018). A Generation Grows Up in China Without Google, Facebook or Twitter. The New York Times. https://nyti.ms/2Lbdl16 


\section{ANEXOS}

\section{ANEXO I \\ Instrumento de recogida de datos
CUESTIONARIO PARA EVALUAR CIBER BULLYNG DEL ALUMNADO DE EDUCACIÓN SECUNDARIA EN LOS CENTROS EDUCATIVOS.

Introducción:

El presente cuestionario tiene como objetivo conocer si existe, bullying virtual en las aulas de alumnos de Educación Secundaria. Los resultados obtenidos se tratarán de forma anónima y confidencial. Esperando obtener sus respuestas con veracidad, se le agradece anticipadamente su valiosa colaboración.

\section{Dimensión Datos demográficos}

Género:

$$
\text { Edad: }
$$

Curso:

- Masculino

1‥E.S.O. $\quad 3$.E.S.O.

- Femenino

$2^{\circ}$.E.S.O. $\quad 4^{\circ}$.E.S.O.

\section{Dimensión Redes Sociales}

P1. ¿Qué redes sociales utilizas?

P2. ¿Cómo es tu perfil en las redes sociales?

PA1. ¿Cómo proteges tus datos personales en las redes sociales?

PA2. ¿Qué tipos de archivos compartes en tus redes sociales?

\section{Dimensión Actitudes en Redes}

E1) Has sufrido agresiones en Facebook

NADA POCO ALGO BASTANTE MUCHO

E2) Has sufrido agresiones en WhatsApp

NADA POCO ALGO BASTANTE MUCHO

E3) Te han etiquetado en fotos comprometedoras

NADA POCO ALGO BASTANTE MUCHO

E4) Te han divulgado rumores en redes sociales

NADA POCO ALGO BASTANTE MUCHO

E5) Te han enviado mensajes ofensivos en redes sociales

NADA POCO ALGO BASTANTE MUCHO

$\begin{array}{lll}\text { E6) Te han enviado mensajes amenazantes en redes sociales } & \\ \text { NADA POCO ALGO BASTANTE } & \text { MUCHO }\end{array}$

E7) Han divulgado tus secretos en redes sociales

NADA POCO ALGO BASTANTE MUCHO

E8) Han violado tu intimidad publicando fotos intimas en redes sociales

NADA POCO ALGO BASTANTE MUCHO

E9) Te han grabado en video para obligarte a hacer algo que no querías.

NADA POCO ALGO BASTANTE MUCHO

E10) Te han excluido de grupos sociales en la red

NADA POCO ALGO BASTANTE MUCHO

E11) Han enviado mensajes maliciosos a otras personas haciéndose pasar por ti.

$\begin{array}{llll}\text { NADA } & \text { POCO } & \text { ALGO }\end{array}$ 


\section{ANEXO II \\ Dimensiones de Instrumento de recogida de datos.}

Estructura del cuestionario en dimensiones (ver tabla 5) para evaluar actitudes nocivas en redes sociales en los estudiantes de secundaria.

Dimensiones del cuestionario.

\begin{tabular}{ll}
\hline DIMENSIONES & ITEMS \\
\hline Dimensión I Datos & Edad \\
demográficos & Género \\
& Etapa
\end{tabular}

Dimensión II Redes Sociales P1. ¿Qué redes sociales utilizas?

P2. ¿Cómo es tu perfil en las redes sociales?

PA1. ¿Cómo proteges tus datos personales en las redes sociales?

PA2 ¿Qué tipos de archivos compartes en tus redes sociales?

\begin{tabular}{ll}
\hline Dimensión III Actitudes en & E1) Has sufrido agresiones en Facebook \\
Redes & E2) Has sufrido agresiones en WhatsApp \\
& E3) Te han etiquetado en fotos comprometedoras \\
E4) Te han divulgado rumores en redes sociales \\
E5) Te han enviado mensajes ofensivos en redes sociales \\
E6) Te han enviado mensajes amenazantes en redes \\
sociales \\
E7) Han divulgado tus secretos en redes sociales \\
E8) Han violado tu intimidad publicando fotos intimas en \\
redes sociales \\
E9) Te han grabado en video para obligarte a hacer algo \\
que no querías. \\
E10) Te han excluido de grupos sociales en la red \\
E11) Han enviado mensajes maliciosos a otras personas \\
haciéndose pasar por ti. \\
\hline PA4 Red que más utilizas con tus amigos \\
"Redes Sociales"
\end{tabular}

\section{INFORMACIÓN SOBRE LOS AUTORES}

Cristina Sánchez Romero es profesora del Departamento de Didáctica, Organización Escolar y Didácticas Especiales de la Facultad de Educación de la UNED. Doctora por la UNED. Máster Oficial en Informática Educativa. Título de Tutor del Espacio Europeo de Educación Superior. Licenciada en Filosofía y Ciencias de la Educación. Cargos Académicos: Vicesecretaria General de Pruebas Presenciales de la UNED. (Actualmente) (2016-2019) Secretaria de la Facultad de Educación. Secretaría Académica y Profesora del Máster/Posgrado Oficial sobre Tratamiento Educativo de la Diversidad (Actualmente). Tutora Centro Asociado de Madrid con Venia Docendi. Entre sus líneas de trabajo destacan las competencias profesionales y digitales, la inclusión educativa y las tecnologías en contextos diversos y vulnerables. ORCID: https://orcid.org/0000-0002-0280-5406 
Maria Carmen López Berlanga es Directora Pedagógica de Educación Infantil y Educación Primaria del Centro López Vicuña de Madrid. Doctora en Educación por la UNED. Licenciada en Psicopedagogía en la especialidad de Educación Especial, Diplomada en Magisterio en la especialidad de educación Primaria, Máster en innovación e investigación en ciencias de la Educación, Máster en Mediación y Orientación Familiar en Educación y Máster en resolución de conflictos en el ámbito educativo. Entre sus líneas de trabajo descata las tecnologias en los contextos ducativos, la convivencia digital en el ambito educativo en cuanto a los riesgos de vulnerabilidad y la inclusión educativa en constextos diversos.

ORCID: https://orcid.org/0000-0002-9375-4834

\section{(c) (i)}

Los textos publicados en esta revista están sujetos a una licencia de Reconocimiento 4.0 España de Creative Commons. Puede copiarlos, distribuirlos, comunicarlos públicamente y hacer obras derivadas siempre que reconozca los créditos de las obras (autoría, nombre de la revista, institución editora) de la manera especificada por los autores o por la revista. La licencia completa se puede consultar en:Licencia Creative Commons Atribución-NoComercial-Compartir por igual 4.0 Internacional. 